\title{
Philosophiques
}

\section{Par-delà le féminisme : pour une éthique sartrienne de la pluralité des points de vue}

\section{Yvan Cloutier}

Volume 21, numéro 2, automne 1994

Les femmes et la société nouvelle

URI : https://id.erudit.org/iderudit/027276ar

DOI : https://doi.org/10.7202/027276ar

Aller au sommaire du numéro

Éditeur(s)

Société de philosophie du Québec

ISSN

0316-2923 (imprimé)

1492-1391 (numérique)

Découvrir la revue

Citer ce document

Cloutier, Y. (1994). Par-delà le féminisme : pour une éthique sartrienne de la pluralité des points de vue. Philosophiques, 21(2), 333-341.

https://doi.org/10.7202/027276ar
Résumé de l'article

Aucun point de vue particulier ou général (racial sexuel, religieux, etc.) ne peut se constituer en éthique. Par contre l'exigence d'universalisation n'implique pas un sujet impersonnel mais se réalise dans le processus d'interpellation des consciences singulières situées et interdépendantes dans leur dévoilement du monde. Comme le montre Sartre, tout projet d'une éhiquedoitreposersurd) l'assomption du fait que tout point de vue soit totalité-détotalisée et sur (2) la reconnaissance de l'interdépendance des consciences. Dès lors, la lucidité doit remplacer la mauvaise foi originelle et la générosité doit faire échec à la tentation de la violence comme refus de l'autre comme liberté. 


\title{
Par-delà le féminisme : pour une éthique sartrienne de la pluralité des points de vue

\author{
par
} Yvan Cloutier
}

\begin{abstract}
RESUME : Aucun point de vue particulier ou général (racial, sexuel, religieux, etc.) ne peut se constituer en éthique. Par contre l'exigence d'universalisation n'implique pas un sujet impersonnel mais se réalise dans le processus d'interpellation des consciences singulières situées et interdépendantes dans leur dévoilement $d u$ monde. Comme le montre Sartre, tout projet d'une éhique doit reposer sur (I) l'assomption du fait que tout point de vue soit totalité-détotalisée et sur (2) la reconnaissance de linterdépendance des consciences. Dès lors, la lucidité doit remplacer la mauvaise foi originelle et la générosité doit faire échec à la tentation de la violence comme refus de l'autre comme liberté.
\end{abstract}

ABSTRACT : No individual or general point of view (racial, sexual, religious, etc.) may constitute itself as an ethic. Nevertheless, the requirement of universalisation does not imply an impersonal subject, but it is lived through the process of interpellation of singular, situated and interdependent consciences. As Sartre claims, an ethic must meet two requirements:(I) to assume the fact that every point of view is totalized-detotalized; and (2) to recognize the interdependency of the consciences. Therefore, lucidity must replace the original bad faith and generosity must counter the temptation of violence as a refusal of the other's freedom.

Nous sommes déjà en mesure d'identifier et d'évaluer la contribution des points de vue des femmes dans les champs littéraire, philosophique et scientifique. Dorénavant plusieurs sciences humaines et la philosophie doivent compter sur les points de vue féminins. La question qui m'intéresse ne concerne pas du point de vue des femmes mais la prétention à constituer une épistémologie ou une éthique à partir de ce point de vue.

L'éthique contemporaine tente de fonder des prescriptions en faisant l'économie de l'exigence de l'universalisation et de toute notion de nature humaine ou de transcendant. Comme nous reconnaissons que nos 
préférences individuelles ne peuvent en rien passer du prudentiel au « devant être fait » (la prescriptibilité), nous sommes dès lors tentés de faire de la communauté géographique, économique ou sexuelle le point d'Archimède sur lequel appuyer les prescriptions morales. A peine croyons-nous être sortis du subjectivisme, que nous nous trouvons aux prises avec le relativisme. Il y aurait alors une éthique africaine et pourquoi pas féministe africaine, féministe africaine « tutsi », une éthique amérindienne, etc. L'ersatz de la communauté nous place cependant devant l'impossibilité de poser des jugements inter-communautaires. Comment justifier l'interdiction des mutilations sexuelles faites aux Africaines ou l'application de la Charte canadienne des droits et libertés aux Amérindiennes et aux Amérindiens, ces derniers la rejetant au nom de leur propre éthique?

Certains éthiciens proposent comme succédané au support de l'universalisation le langage moral, la psychologie morale ou la sociobiologie; d'autres, tels Rawls et Habermas, explorent des approches plutôt procédurales, tels l'« équilibre réflexif élargi » ou le consentement des agents potentiellement impliqués en tant que participants à un discours pratique. L'éthique à laquelle travailla Sartre dans les années 40, et que nous connaissons grâce aux inédits publiés récemment, me semble susceptible d'alimenter notre recherche. En effet l'analyse sartrienne, d'emblee antifondationaliste, part des points de vue des individus pour déboucher étrangement sur un objectivisme éthique. En bref, pour Sartre, il n'y a que des points de vue mais aucun des points de vue ne peut se poser comme totalisant. La pluralité des points de vue n'aboutit pas à un quelconque relativisme bienveillant mais à une solidarité fondée sur l'interdépendance des consciences. La vulnérabilité des consciences rend possible la violence et l'exploitation mais aussi la générosité et la solidarité. Cette approche me semble susceptible de nous aider à penser les rapports que les hommes et les femmes doivent entretenir face à leurs points de vue respectifs.

Dans un premier temps, je présenterai le plus brièvement et le plus simplement possible les tenants et aboutissants de l'argumentation de Sartre. Dans un deuxième temps, je l'appliquerai à la question des dites éthiques communautaires et, plus spécifiquement, à l'interdépendance des points de vue des hommes et des femmes.

\subsection{1 n'y a que des points de vue individuels}

\section{De l'interdépendance à la générosité}

Sur la base de ses études de psychologie phénoménologique inspirées par la phénoménologie husserlienne, Sartre décrit la conscience comme une structure relationnelle. Toute conscience est conscience de quelque chose et elle n'est pas ce dont elle est conscience. Perceptions, objet irréel imaginé, valeurs, voire même l'ego, sont des objets transcendants produits par une conscience située (temporalisée, incarnée, sexuée, etc.). Les représentations ne sont pas le simple reflet du monde mais réextériorisation d'une intériorisation. 
La structure dévoilante de la conscience interdit tout point de vue impersonnel ou tout appel à un observateur impartial; cela « signifie, écrit Sartre, que je dois renoncer à cette tendance perpétuelle à glisser entre mon point de vue et la réalité les lunettes de l'universel, ou plutôt de l'absence de tout point de vue » (Cahiers pour une morale 503; dorénavant CPM). Cet individualisme épistémologique exclut tout recours à des entités supra-individuelles, tels l'Humanité, l'Esprit. Il n'y a pas, écrit Sartre, « un principe d'unité réelle derrière les consciences individuelles et les collectivités particulières » (CPM II3). Il n'y a que nos dévoilements. Et c'est en ce sens qu'il faut entendre la notion d'humanisme et son corollaire, la responsabilité sociale. Pour reprendre la formulation des Cahiers, l'homme est l'être par lequel la valeur vient au monde.

Les conséquences de cet argument sont décisives : (I) refus de tout naturalisme éthique; (2) l'historicité des valeurs; (3) les prescriptions éthiques commencent par le moment subjectif, celui de la lucidité; (4) ma responsabilité comme co-producteur de valeurs (solidarité).

L'exigence première de l'éthique sartrienne devient la lucidité. Chaque conscience singulière doit assumer sa responsabilité de productrice de valeurs et ne pas abdiquer dans la mauvaise foi en s'en remettant à un ordre des choses ou à la simple rationalité. Ce moment subjectif est inéluctable et constitue le prérequis du deuxième moment qui, lui, est intersubjectif et met en relation les producteurs de valeurs. Dès lors toute valeur devient proposition à une autre liberté et non référence à un ordre qui s'imposerait d'emblée aux consciences singulières. C'est ici que Sartre réintègre un principe d'universalisation qui désigne l'humanité virtuelle ${ }^{I}$, c'est-à-dire les consciences qui auront à reprendre ma proposition.

\subsection{Chaque point de vue est une totalitié-détotalisée}

Si toute valeur est le produit d'un point de vue singulier, elle est toujours le corrélat d'une conscience temporalisée et située (sexuellement, socialement, etc.), et par conséquent elle est, pour reprendre la formulation de Sartre, une totalité-détotalisée. C'est-à-dire que ce tout qu'est mon jugement de valeur en un temps Tr, est susceptible d'être repris par ma conscience en un T2, et ainsi de suite en T3..., T4, etc.

En outre, cette production acquiert un second caractère d'objectivité2 ${ }^{2}$ par les autres consciences singulières. Pour reprendre le jargon sartrien, la valeur comme en-soi pour-soi devient en-soi-pour-soi pour autrui et en-soi-pour-soi par autrui. Prenons l'exemple d'une œuvre littéraire : l'auteur produit ce qui pour lui est l'œuvre I (OI); cette œuvre lue par un lecteur devient O.I.2, cet O.I.2

I. « Il y a des actions dont la maxime suppose l'universel et, par conséquent, qui se réfèrent à l'humanité virtuelle [...] » (CPM 442).

2. «L'objectivité de ma situation c'est ma situation pour l' Autre. » (CPM 470). 
est perçu par l'auteur comme O.I.2.I, et ainsi de suite. L'infinité des points de vue interdit toute totalisation d'enveloppement; ainsi « llel génie de Proust, écrit Sartre (L'Être et le néant I4), même réduit aux œuvres produites, n'en équivaut pas moins à l'infinité des points de vue possibles qu'on pourra prendre sur cette œuvre et qu'on nommera "l'inépuisabilité" de l'œuvre proustienne ».

La réception de cette œuvre forme un amalgame, Sartre parlerait d'une totalité d'extériorité dans laquelle ni les lecteurs singuliers ni l'auteur ne se reconnaissent. Dans les inédits des années 6o, et en particulier dans la conférence de Rome (1964), Sartre applique à l'analyse des valeurs la notion de pratico-inerte qu'il avait développée dans la Critique de la raison dialectique. La norme éthique n'échappe pas à la réification de la praxis, elle se présente comme « inerte détermination de la praxis par l'autre ». Comme Simone de Beauvoir l'avait déjà montré dans Pour une morale de l'ambiguité (1947) à propos de la musulmane et de l'esclave, le processus de reproduction bloque la compréhension véridique des situations. Beauvoir et Sartre analyseront la genèse de ces antivaleurs, et c'est l'éthique qui commandera le renversement de valeurs établies et posées comme s'imposant aux individus. Les représentations des rôles masculins et féminins comme totalités constituées ont une efficace sur l'appropriation par chaque homme et chaque femme de sa condition sexuée; on bloque alors le moment subjectif, celui de la lucidité, qui est la condition nécessaire au choix que je ferai de moi considérant les divers possibles.

\section{3 l'interdépendance des points de vue}

Le lecteur révèle à l'auteur un point de vue sur l'œuvre que l'auteur ne peut avoir par lui-même. De même, je ne puis avoir l'expérience de mon dos (vu de l'extérieur); ici l'autre enrichit ma compréhension du monde, comme le décrit Sartre:

IVloir un homme de dos, c'est le voir à partir de ce qu'il ne voit pas, c'est le constituer à partir de ce qu'il ignore, c'est prévoir ce qu'il ne peut prévoir et le prévoir lui-même à partir de ce qu'il ne prévoit pas; voir la pierre qu'il ne voit pas rouler sur lui, c'est précisément se faire dévoiler à partir de la pierre son être-au-milieu-du-monde comme possibilité permanente et reçue. donnée de ne plus y être l...] (CPM 517).

Le rapport d'interdépendance peut enrichir la compréhension de ma situation comme il peut me mettre en péril, dépendamment du choix que fera l'autre de me reconnaître dans ma liberté :

Ainsi par l'Autre je suis enrichi d'une nouvelle dimension de l'Être, par l'Autre je me mets à exister dans la dimension de l'Être, par l'Autre je deviens objet. Et ceci n'est nullement une déchéance ou un péril en soi. Cela ne le deviendra que si l'Autre refuse de voir aussi en moi une liberté. Mais si au contraire il me fait exister comme liberté existant aussi comme Être-objet, s'il fait exister comme moment autonome et thématisé cette contingence que je dépasse perpétuellement, il enrichit le monde et moi-même, il donne un sens à mon existence en plus du sens subjectif que je lui donne moi-même [...] (CPM 515). 
L'auteur ne peut échapper à ce risque d'être lu rapidement et d'être mis en boîte par le lecteur. Par contre, le lecteur généreux enrichit ma compréhension de l'œuvre.

La possibilité de l'erreur et le fait que la consience de l'erreur passe souvent par l'autre confirment le rapport d'interdépendance entre les consciences. Un point de vue spécifique peut manquer certains aspects du réel ou le déformer. Les déformations, telles les illusions perceptuelles, les représentations substitutives et les hallucinations, échappent au sujet à moins qu'il n'en ait la révélation par un autre sujet. La conscience de l'erreur vient par l'autre, dont le point de vue devient essentiel à la réalisation de mon projet, comme le montre Sartre à propos de deux camarades de combat :

Japprouve ce camarade de combat que je vois de loin ramper vers l'ennemi, je me bats comme lui, son projet est mien. Mais je vois qu'il donne tête baissée dans un piège. L'origine de sa conduite c'est l'ignorance et je suis pareillement ignorant en ce qui me concerne. Mais cette ignorance qui n'est rien quand je vis mon action, je la dévoile chez l'autre. Le résultat : je dévoile son projet comme se détruisant lui-même. (CPM 519)

Dans les années i950 et 196o, la politisation de la pensée sartrienne l'amènera à mettre au réfrigérateur cette morale ontologique, pour penser davantage la constitution des valeurs et le conditionnement des consciences ${ }^{3}$ dans le champ de la rareté. Aux yeux de Sartre, dans un monde marqué par la pénurie et par l'exploitation, la solidarité du groupe s'impose. La réalisation de mes possibles passe par la qualité du dévoilement de l'autre et par sa générosité. Les agents se retrouvent comme ces quatre personnes obligées de défendre une maison assiégée par un ennemi; le meilleur état du monde sera pour elles celui où chacun sera conscient de sa responsabilité de dévoilant visà-vis des autres. Selon nous, Sartre n'abandonne pas sa morale ontologique, contrairement à ce que suggère une remarque rapportée par Simone de Beauvoir dans La Force de l'âge ${ }^{4}$. Dans les Cahiers pour une morale, Sartre donne de la chair à la formule vide «je suis obligé de vouloir en même temps que ma liberté la liberté des autres » de L'Existentialisme est un humanisme (83). La morale ontologique ne renvoie-t-elle pas à un monde qui n'existe pas encore? Que faire de celui qui refuse de me reconnaître comme liberté ? La violence n'estelle pas une médiation nécessaire pour parvenir à un monde dans lequel l'abolition de la rareté rendra possible la suppression de l'exploitation ? Entretemps, y a-t-il des exigences amorales (ou anté-morales) de groupes ou de classes ? L'éthique fonctionne dès lors à l'utopie comme l'illustre les

3. Dès les années I940, Simone de Beauvoir décrivait ces situations bloquées.

4. «Sa morale proprement dite. Sartre l'abandonna cette année-là parce qu'il se convainquit que l' 'attitude morale apparaît quand les conditions techniques et sociales rendent impossibles les conduites positives. La morale, c'est un ensemble de trucs idéalistes pour vous aider à vivre ce que la pénurie des ressources et la carence des techniques vous imposent" (notes inédites) » (p. 277). 
descriptions de l'Apocalypse dans les Cahiers, et du groupe en fusion dans la Critique.

\subsection{Une morale de la lucidité et de la générosité}

La vulnérabilité générée par cette interdépendance peut mener tant à la violence et à l'exploitation qu'à la reconnaissance et à la générosité. La relation parentale, le rapport pédagogique et la séduction ont la violence comme un de leurs posssibles. Pour Sartre, la violence consiste à couper l'autre de sa fin et à l'inclure dans sa propre finalité de mère, de professeur ou d'amant. Au lieu d'agir de manière à ce que la liberté de l'autre s'exerce dans une conscience lucide et véridique de sa situation, je piège l'autre de manière à ce qu'il consente à prendre mes fins comme les siennes.

Je puis discréditer le point de vue d'une adolescente en lui disant qu'elle n'est pas encore à même de comprendre que tel choix s'impose à elle, d'une femme en l'amenant à croire que le respect de la vie humaine passe avant son projet de femme amoureuse, professionnelle, etc. De même je puis discréditer le point de vue d'un Africain ou d'une Africaine croyant à la nécessité de la mutilation des femmes. Ne pourrais-je pas les reconnaittre comme des interlocuteurs égaux qui, comme moi, véhiculent des croyances rationnellement non justifiées ? Peut-être m'apprendront-ils des choses sur le respect dû aux anciens et sur la qualité du tissu humain. Dévaloriser la capacité réflexive de l'individu l'enracine davantage dans l'esprit de sérieux de sa culture enfantine, sexiste ou ethnique. Voilà pourquoi Sartre, qui pense l'éthique avec le paradigme de l'œuvre d'art, place la générosité au sommet de la hiérarchie des valeurs ( (CPM 486; 509-510).

Ainsi l'éthique sartrienne des années 1940 repose sur deux valeurs : la lucidité et la générosité, la première déterminant le rapport à soi comme créateur de valeurs, et la seconde régissant la relation aux autres consciences.

\section{Des points de vue individuels sexués}

L'argumentation de Sartre est somme toute très simple, voire même simpliste. Mais quelles en sont les conséquences si on les applique à notre question des points de vue communautaires, et plus spécifiquement des points de vue des femmes et des hommes?

\subsection{Reconnaitre la « sexuation » des points de vue singuliers}

La première exigence consiste à assumer que les valeurs soient des corrélats de points de vue singuliers et conditionnés par la situation de ces consciences. Chaque conscience réextériorise les conditionnements sociaux qu'elle a intériorisés. Dès lors l'important n'est pas ce que l'on a fait de Genet ou de Flaubert, mais ce qu'ils ont fait à partir de ce qu'on avait fait d'eux; pour reprendre la terminologie de L'Idiot de la famille, la personnalisation ne se réduit pas à la constitution de la personne.

Il en est de même pour la situation en tant que « sexuée». Conditionnements psychophysiologiques et représentations sont intériorisés 
et réextériorisés. Il en résulte que, de même qu'il n'y a pas de point de vue impersonnel mais des productions impersonnelles, de même il ne saurait y avoir de point de vue masculin ou féminin mais des reproductions amalgamées à partir des dévoilements singuliers.

Le processus de reproduction idéologique, qui méconnaît la singularité des visées et impose une totalité constituée et fermée, implique une violence, car on y coupe la personne de tout rapport des représentations-moyens à ses propres fins.

\section{2 les gains de la pluralité des points de vue}

Dès lors chaque femme et chaque homme a un point de vue sur ce qu'on a fait de lui ou d'elle comme homme ou femme. Assumer la relativité de son dévoilement, c'est en reconnaître le caractère partiel et ouvrir à l'élargissement de sa compréhension du monde et de soi grâce aux points de vue des autres.

Historiquement, la reconnaissance et la valorisation des visées singulières ont emprunté la médiation de la classe ou du groupe. Les femmes ont reconnu la légitimité de leur point de vue, de leur parole, en se parlant entre elles; il en fut de même pour la négritude et la classe ouvrière. Toutefois cette parole solidaire doit beaucoup aux brèches, à des points de vue d'emblée marginaux : pensons à Simone de Beauvoir, à Wilhem Reich et à Senghor.

Récemment ${ }^{5}$ des historiennes italiennes faisaient le bilan de leur contribution non seulement au domaine de l'histoire faite par des femmes mais aussi à l'évolution de l'ensemble du champ des études historiques. Les historiens et les historiennes œuvrent maintenant dans un espace symbolique qui prend en compte le travail des historiennes. Le procès Kennedy-Smith est intéressant en ce qu'il a mis en cause la représentation-amalgame de la sexualité dans son paradigme de la pénétration. Encore ici la déconstruction d'une telle représentation instituée passe par la reconnaissance de points de vue différents; la pénétration est un possible.

La vérité passe ici par l'autre mais encore faut-il accueillir une information qui risque de fissurer une représentation constituée du monde.

\subsection{1 le « qui perd gagne ! »}

La thèse selon laquelle la conscience véridique de soi et du monde passe par la lucidité m'apparaît comme un apport majeur de la philosophie sartrienne. La psychanalyse ne saurait se réduire à un mécanicisme et la libération au seul changement de conditions objectives.

La résistance à reconnaître les conditionnements singuliers et par conséquent sexués des représentations vient des enjeux non seulement inconscients mais de ce que Sartre appelle l'insécurité ontologique. Pour

5. Discutendo di storia. Soggetivitá, ricerca, biografia, a cura della Società Italiana delle Storiche, Rosenberg et Sellier, Torino, 1990. 
Sartre, les individus se constituent dès l'enfance une représentation du monde sécurisante dans laquelle les éléments s'imbriquent. Toute l'expérience ultérieure s'organisera autour de cette représentation fondatrice ${ }^{6}$. La vie psychique est pour Sartre, comme pour Freud, unifiée; mon attitude face à une montagne, à un examen, à une expérience amoureuse, à mes pratiques sportives, s'organise autour d'un même noyau. Une étude menée par des chercheurs de l'Estrie sur les syndromes de sevrage des coureurs à pied de longues distances illustre bien la thèse sartrienne. Dans un premier temps, on est tenté d'expliquer les symptômes par le fait que l'organisme ne produise plus d'endorphine. Mais les pianistes contraints d'interrompre leur activité développent les mêmes symptômes. Ne serait-ce pas que les coureurs et les pianistes se retrouvent privés d'un point de repère important qui fissure la représentation de leur identité? N'en serait-il pas de même dans le rapport aux représentations ? L'insécurité ontologique favorise les conduites de récupération, de séduction et de domination, qui sont trois conduites de violence. Le conditionnement idéologique a ses racines psychologiques et l'ouverture aux points de vue de l'autre sexe implique, et ce davantage pour les hommes dont l'identité sexuelle est plus fragile et par conséquent plus rigide, l'acceptation de l'insécurité ontologique. C'est le « qui perd gagne ».

\section{Conclusion}

L'analyse sartrienne de la conscience et la thèse de l'individualisme épistémologique que nous en avons dégagée, nous ont fourni un point d'Archimède à partir duquel nous croyons pouvoir proposer des critères de conduite acceptables eu égard aux points de vue des hommes et des femmes. Discréditer le point de vue féminin peut avoir les mêmes effets que le discrédit par des féministes «séparatistes » des points de vue des hommes, points de vue qu'il ne faut pas confondre avec leurs productions.

D'un point de vue sociologique, la revendication d'une pensée et d'une éthique au féminin est acceptable : il existe de ces totalités (amalgamées) de représentations. D'un point de vue prudentiel, pour ne pas dire militant, cette revendication a eu pour effet de confirmer les femmes dans leur pouvoir représentationnel et d'établir un rapport de force. Le féminisme a eu ici et aura toujours son rôle de contribution à l'émergence et à la reconnaissance des points de vue des femmes d'abord entre elles et ensuite par les hommes. Mais d'un point de vue éthique, cette prétention à une épistémologie ou à une éthique féministes se détruit en se posant parce qu'elle pose une totalité fermée.

Mais peut-être ne sommes-nous pas encore dans les conditions qui rendent possible l'expérience éthique. Se posent alors les questions de la priorité du point de vue moral sur les autres points de vue, et de l'acceptabilité

6. Je renvoie les lecteurs et lectrices aux psychanalyses existentielles que Sartre a faites de Flaubert, de Baudelaire, de Jean Genet et de Sartre lui-même (Les Mots). 
d'une morale de groupe comme moment vers une éthique englobant la pluralité des points de vue. L'éthique fonctionne ici à l'utopie et fournit une idée régulatrice permettant de justifier des actes qui apparaîtront comme des stases si on ne les comprend pas comme des moments vers une fin qui soit la reconnaissance généralisée des points de vue.

\section{Références}

BEAUVOIR, Simone de, La Force de lâge. Tome I. Paris, Gallimard, « Le livre de poche $\gg, 1963$.

BEAUVOIR, Simone de, Pour une morale de l'ambiguité, suivi de Pyrrhus et Cinéas, Paris, Gallimard, « Idées », 1947.

SARTRE, Jean-Paul, Les Cahiers pour une morale, Paris, Gallimard, 1983.

SARTRE, Jean-Paul, La Critique de la raison dialectique, précédé de Questions de méthode, Tome I, Théorie des ensembles pratiques, Paris, Gallimard, 1985.

SARTRE, Jean-Paul, L'Existentialisme est un humanisme, Paris, Nagel, 1966 lvolume paru en 19466 . 